J. Clin. Chem. Clin. Biochem.

Vol. 18, 1980, pp. 909-915

\title{
Der Einfluß des Funktionszustands der Schilddrüse auf den Anstieg des cyclischen AMP im Plasma nach Glukagoninjektion
}

\author{
Von W. Jäckel, J. Heimes und J. Herrmann
}

Medizinische Klinik C(Direktor: Prof. Dr. H. L. Krüskemper), Universität Düsseldorf

(Eingegangen am 14. Januar/7. Juli 1980)

Zusammenfassung: Zur Beantwortung der Frage, in welchem Maße der Anstieg des cyclischen AMP im Plasma nach Glukagon-Injektion beim Menschen durch den Funktionszustand der Schilddrüse beeinflußt wird, wurden 26 Normalpersonen, 36 Patienten mit einer Hyper-, 35 mit einer Hypothyreose sowie 24 Patienten mit blander Struma untersucht.

Allen Testpersonen wurde in nüchternem Zustand nach vorheriger Blutentnahme $1 \mathrm{mg}$ Glukagon in die Cubitalvene injiziert und 15 Minuten später erneut $5 \mathrm{ml}$ Blut zur Bestimmung der cyclischen AMP-Konzentration entnommen. Die Messung des cAMP erfolgte als Doppelbestimmung in einem Protein-Bindungs-Assay. Nach Glukagon-Stimulation wurde bei Normalpersonen eine mittlere cAMP-Konzentration von $483 \mathrm{nmol} / 1$ gefunden. Hypothyreote Patienten wiesen nach Glukagon-Injektion eine mittlere cAMP-Konzentration von nur 229 nmol/l auf. Bei hyperthyreoten Patienten stieg das cAMP im Plasma im Mittel auf $871 \mathrm{nmol} / 1$. Die Unterschiede der mittleren cAMP-Konzentrationen nach Glukagon-Injektion sind zwischen den drei Kollektiven hoch signifikant.

Unter Substitutionstherapie mit $L$-Thyroxin bei 7 hypothyreoten Patienten sowie nach thyreostatischer Therapie bei 18 hyperthyreoten Patienten normalisierten sich die glukagonstimulierten cAMP-Konzentrationen.

4 Patienten mit dekompensierter Lebercirrhose zeigten stark erniedrigte cAMP-Werte 15 Minuten nach GlukagonGabe, während ein Patient mit Verschlußikterus eine deutlich erhöhte Reaktion aufwies.

Der Glukagontest stellt u. E. unter Berücksichtigung der durch schwere Leber- und Gallenwegserkrankungen beeinflußten Resultate eine sinnvolle zusätzliche Kenngröße zur Beschreibung der schilddrüsenabhängigen Stoffwechselsituation in der Peripherie dar. Er kann aber die Bestimmung von $T_{3}$ und $T_{4}$ sowie den Thyroliberin (TRH)-Test in der Schilddrüsen-Diagnostik wegen mangelnder Spezifität nicht ersetzen.

\section{The effect of thyroid function on the increase of plasma cyclic AMP following glucagon injection}

Summary: The increase in plasma cyclic adenosine- $3^{\prime}: 5^{\prime}$-monophosphate (cAMP) was measured after intravenous injection of $1 \mathrm{mg}$ of glucagon in 26 normal subjects, 36 patients with hyperthyroidism, 35 patients with hypothyroidism and 24 patients with euthyroid goitre.

While patients with euthyroid goitre responded normally, the plasma cyclic AMP response in patients with hyperthyroidism was considerably increased and in those with hypothyroidism decreased. 4 patients with cirrhosis of the liver had reduced responses and 1 patient with extrahepatic obstructive jaundice an enhanced response.

This test seems to be a valuable additional parameter for the description of the thyroid-dependent metabolic situation. However, because of its unspecificity it cannot replace the measurement of serum $T_{3}, T_{4}$ and thyrotropin (TSH) response to thyroliberin (TRH).

\section{Einleitung}

Seit Rall \& Sutherland 1957 (1) erstmals die Rolle des Adenosin $-3^{\prime}: 5^{\prime}$-monophosphat (cyclisches AMP) bei der Phosphorylaseaktivierung beschrieben hattèn, ist eine Vielzahl von Arbeiten über die Bedeutung des CAMP $^{1}$ ) erschienen, und inzwischen hat die Bestimmung des
cAMP einen festen Platz in der Diagnostik u. a. des Hypoparathyreoidismus.

\footnotetext{
1) Abkürzungen:

cAMP = cyclisches Adenosin-3': $5^{\prime}$-monophosphat

$\mathrm{T}_{4}=L$-Thyroxin

$T_{3}=L$-Triiodthyronin
} 
Uber den Einfluß von Glukagon auf das cAMP-System berichteten zuerst Sutherland et al. 1966 (2). 1970 konnten Broadus et al. (3) einen Anstieg der Konzentration von CAMP im Plasma nach Glukagoninjektion beim Menschen nachweisen. 1973 zeigten Campbell et al. (4), daß der glukagoninduzierte cAMP-Anstieg in Rattenleberorgankulturen durch Präinkubation mit Schilddrüsenhormonen erheblich verstärkt werden konnte. Die daraufhin naheliegende Frage, ob der cAMP-Anstieg im Plasma des Menschen nach Glukagoninjektion möglicherweise auch durch den Funktionszustand der Schilddrüse beeinflußt wird, untersuchten Elkeles et al. 1974 (5). Ihre Untersuchungsergebnisse an 9 hyperthyreoten und 5 hypothyreoten sowie 10 euthyreoten Patienten zeigten einen deutlichen Unterschied im cAMP-Anstieg der untersuchten Kollektive. In der Folgezeit erschienen mehrere - teilweise widersprüchliche - Arbeiten zu der gleichen Fragestellung (6-9).

Eine exakte Untersuchung des Einflusses des Funktionszustands der Schilddrüse auf den glukagoninduzierten cAMP-Anstieg ist nicht nur von theoretisch-physiologischem, sondern auch von praktischem Interesse, da diesem Glukagontest möglicherweise eine Bedeutung in der Diagnostik von Hyper- und Hypothyreose zukommen könnte.

Unter Hyperthyreosen werden - nach der Definition der Deutschen Gesellschaft für Endokrinologie - Krankheitsbilder verstanden, bei denen in der Peripherie ein Überschuß an Schilddrüsenhormon vorliegt. Entsprechend ist eine Hypothyreose durch einen Mangel an Schilddrüsenhormon in der Peripherie gekennzeichnet. Unter Peripherie sollten im strikten Sinne die hormonsensitiven peripheren Gewebe aufgefaßt werden, in denen beim Menschen der Hormonüberschuß bzw. -mangel nicht direkt nachgewiesen werden kann. In diesem Sinne stellen Hormonkonzentrationen im Blut lediglich indirekte Größen zur Beurteilung des Funktionszustands der Schilddrüse dar. Aus diesem Grunde wird z.Zt. intensiv nach einem praktikablen und verläßlichen Verfahren gesucht, das, wie der Grundumsatz, Rückschlüsse auf die Wirkung von Schilddrüsenhormon an den peripheren Geweben ermöglicht. Mit den hier vorgelegten Untersuchungen soll u.a. die Frage beantwortet werden, ob der sog. Glukagontest ein solches Verfahren darstellt.

\section{Methodik}

\section{Patienten}

Untersucht wurden 36 Patienten mit einer Hyperthyreose, 35 Patienten mit einer Hypothyreose, 24 Patienten mit einer blanden Struma und 26 Normalpersonen. Neben Anamnese und klinischem Befund wurden zur Diagnostik von Hyper- und Hypothyreose noch das routinemäßig bestimmte $T_{3}, T_{4}$ und Thyrotropin (TSH) im Serum herangezogen. Bei allen Patienten lag außerdem ein Schilddrüsenszintigramm vor. Patienten und untersuchte Normalpersonen wurden über die Art der Untersuchung vorher informiert und hatten ihr Einverständnis erklärt.
Von den hyperthyreoten Patienten waren 32 weiblich und 4 männlich, im Alter zwischen 15 und 73 Jahren (Durchschnittsalter: $48 \mathrm{Jahre}$ ). Das mittlere $\mathrm{T}_{3}$ im Serum lag bei $5370 \pm$ $\left.2390 \mathrm{ng} / 1^{2}\right)(\overline{\mathrm{x}} \pm \mathrm{s})$ (Normalbereich 600-2000 ng/l). Die mittlere $\mathrm{T}_{4}$-Konzentration in dieser Gruppe wurde mit $172 \pm 72 \mu \mathrm{g} / \mathrm{l}$ bestimmt (Normalbereich 50-140 $\mu \mathrm{g} / \mathrm{l}$ ). Die hohe Standardabweichung der $\mathrm{T}_{4}$-Werte bei hyperthyreoten Patienten ist dadurch erklärt, daß ein Teil dieser Gruppe durch isolierte $T_{3^{-}}$ Hyperthyreosen repräsentiert wird. Das Thyrotropin lag bei 1,5 $\pm 0,9 \mathrm{mU} / 1$ (obere Normgrenze: $4 \mathrm{mU} / 1$ ).

26 der hypothyreoten Patienten waren weiblich und 9 männlich. Das Alter lag zwischen 18 und 85 Jahren (Durchschnittsalter: $46 \mathrm{Jahre}$ ). In dieser Gruppe wurde ein mittlerer $\mathrm{T}_{3}$-Wert von $640 \pm 530 \mathrm{ng} / \mathrm{l}$, ein $\mathrm{T}_{4}$ von $27 \pm 19 \mu \mathrm{g} / \mathrm{l}$ und ein Thyrotropin von $30,0 \pm 13,9 \mathrm{mU} / 1$ gemessen.

Als Normalpersonen wurden 16 Frauen und 10 Männer im Alter zwischen 22 und 71 Jahren (Durchschnittsalter: 45 Jahre) untersucht. Folgende Mittelwerte bzw. Standardabweichungen wurden ermittelt: $T_{3} 1360 \pm 190 \mathrm{ng} / 1, T_{4} 81 \pm 24 \mu \mathrm{g} / 1$ und Thyrotropin 1,4 $\pm 0,9 \mathrm{mU} / 1$. Der fehlende Unterschied der gemessenen Thyrotropin-Werte bei den Normalpersonen und den hyperthyreoten Patienten spiegelt die bekannte Unsicherheit des Thyrotropin-Radioimmunoassay im unteren Meßbereich wider.

Von den untersuchten Patienten mit einer blanden Struma waren 17 Frauen und 7 Männer im Alter zwischen 20 und 74 Jahren (Durchschnittsalter: 45 Jahre). Hier lag das $T_{3}$ bei $1550 \pm 550 \mathrm{ng} / \mathrm{l}$, das $\mathrm{T}_{4}$ bei $84 \pm 24 \mu \mathrm{g} / \mathrm{l}$ und das Thyrotropin bei $1,6 \pm 1,1 \mathrm{mU} / 1$.

Der Glukagontest wurde an 18 hyper- und 7 hypothyreoten Patienten, die nach Behandlung sowohl klinisch als auch laborchemisch eine normale Schilddrüsenstoffwechsellage aufwiesen, wiederholt. Das $T_{3}$ der ehemals hyperthyreoten Patienten lag nach Kompensation des Funktionszustands bei $1400 \pm 440 \mathrm{ng} / 1$ und das $\mathrm{T}_{4}$ bei $88 \pm 41 \mu \mathrm{g} / \mathrm{l}$. Bei den vorher hypothyreoten Patienten wurde nach der Behandlung mit Schilddrüssenhormon ein $\mathrm{T}_{3}$ von $1340 \pm 400 \mathrm{ng} / \mathrm{l}$ und ein $\mathrm{T}_{4}$ von $96 \pm 30 \mu \mathrm{g} / \mathrm{l}$ gemessen.

\section{Versuchsablauf}

Den Normalpersonen und den Patienten mit Schilddrüsenerkrankungen wurde in nüchternem Zustand Blut aus der Cubitalvene zur Bestimmung der basalen cAMP-Konzentration, des $T_{3}, T_{4}$ und des Thyrotropin entnommen. Danach wurden $1 \cdot \mathrm{mg}$ Glukagon intravenös injiziert und 15 Minuten später erneut $5 \mathrm{ml}$ Blut zur Bestimmung der cAMP-Konzentration entnommen.

Das Blut wurde sofort nach der Entnahme in vorbereitete Röhrchen mit $50 \mu 1$ 0,5 mol/1 EDTA-Lösung (zur Hemmung der Phosphodiesterase) gefüllt und zentrifugiert, das Plasma bis zur Bestimmung des CAMP bei $-20^{\circ} \mathrm{C}$ aufbewahrt.

\section{Bestimmung}

Die Bestimmung des cAMP erfolgte durch einen kommerziellen Radioliganden-Bindungsassay der Firma Amersham.

Um im optimalen Bereich der Standardkurve ablesen zu können, wurden die 15 Minuten nach Glukagoninjektion gewonnenen Seren 1:3 mit Pufferlösung verdünnt. Die Inter- und Intraassayvarianz beträgt bei diesem Proteinbindungsassay unter $7 \%$.

Die Signifikanz zwischen den einzelnen Kollektiven bezüglich des CAMP-Anstiegs 15 Minuten nach Glukagon-Injektion wurde mittels des t-Testes nach Student ermittelt.

\section{Ergebnisse}

Die basalen Konzentrationen des cAMP bei 26 Normalpersonen, 35 Hypo-, 36 Hyperthyreosen und 24 Patien-

2) Umrechnungsfaktoren:

$\mathrm{T}_{3}: \mathrm{ng} / 1 \times 0,00154=\mathrm{nmol} / 1$

$\mathrm{T}_{4}: \mu \mathrm{g} / \mathrm{l} \times 1,28=\mathrm{nmol} / \mathrm{l}$. 
ten mit blander Struma sind in Abbildung 1 dargestellt. Zwischen den Euthyreosen $(12 \pm 9 \mathrm{nmol} / \mathrm{l})$ und den Hypothyreosen $(10 \pm 5 \mathrm{nmol} / \mathrm{l})$ zeigte sich kein wesentlicher Unterschied. Der Mittelwert bei den hyperthyreoten Patienten lag zwar deutlich höher (25 \pm $12 \mathrm{nmol} / \mathrm{l})$, jedoch bestanden zwischen der letzteren Gruppe und der der Normalpersonen große Uberschneidungen.

In Abbildung 2 ist der Verlauf der cAMP-Konzentrationen nach Glukagon-Stimulation aufgezeichnet. Wie zu sehen, ergab sich nach 15 Minuten eine Konzentrationsspitze mit den deutlichsten Differenzen zwischen den einzelnen Kollektiven.

Die zu diesem Zeitpunkt in größeren Gruppen gemessenen Einzelwerte sind zusammen mit den entsprechenden Mittelwerten und Standardabweichungen in Abbildung 3 dargestellt.

In allen Kollektiven wurde ein deutlicher Anstieg der cAMP-Konzentration festgestellt. Dieser war jedoch bei den Hyperthyreoten $(871 \pm 199 \mathrm{nmol} / \mathrm{l})$ signifikant größer als bei den Normalpersonen ( $483 \pm 66 \mathrm{nmol} / \mathrm{l})$. Ubberlappungen der Einzelwerte zwischen diesen Bereichen traten nicht auf. Bei den hypothyreoten Patienten wiederum war der cAMP-Anstieg gegenüber dem Normalkollektiv deutlich erniedrigt $(229 \pm 91 \mathrm{nmol} / \mathrm{l})$. Zwei der hypothyreoten Patienten hatten allerdings trotz deutlich hypothyreoter Stoffwechsellage $\left(\mathrm{T}_{3}: 70 \mathrm{bzw}\right.$. $\left.260 \mathrm{ng} / \mathrm{l} ; \mathrm{T}_{4} 7 \mathrm{bzw} .6 \mu \mathrm{g} / \mathrm{l}\right)$ nach 15 Minuten einen cAMP-Anstieg, der dem des Normalkollektivs vergleichbar war (436 bzw. $407 \mathrm{nmol} / \mathrm{l})$ (Abb. 3).

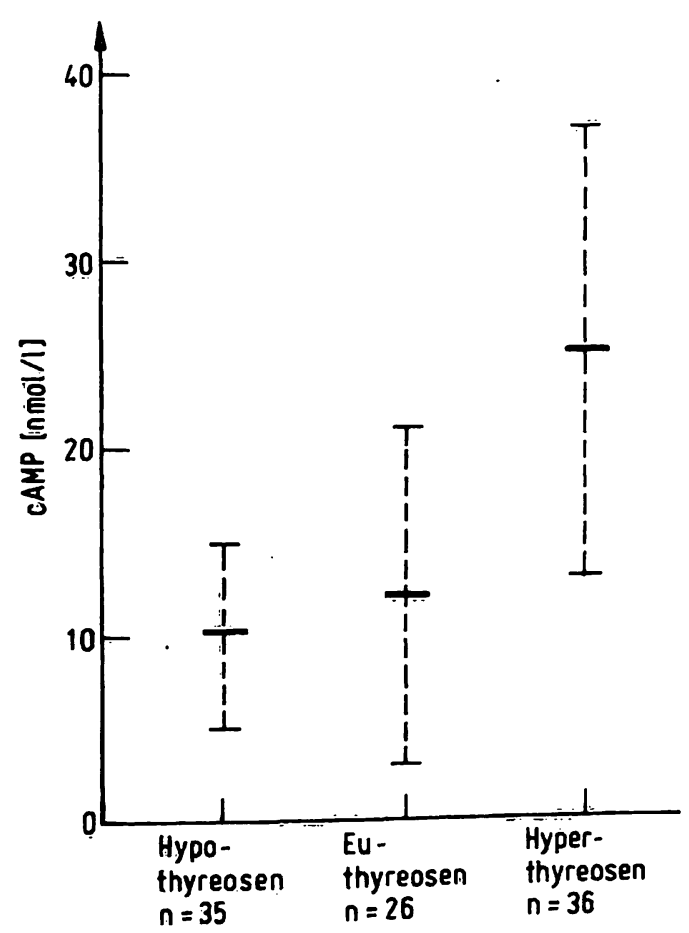

Abb. 1. Basale Konzentrationen des cAMP im Plasma bei Normalpersonen und bei hypo- und hyperthyreoten Patien$\operatorname{ten}(\bar{x} \pm s)$.

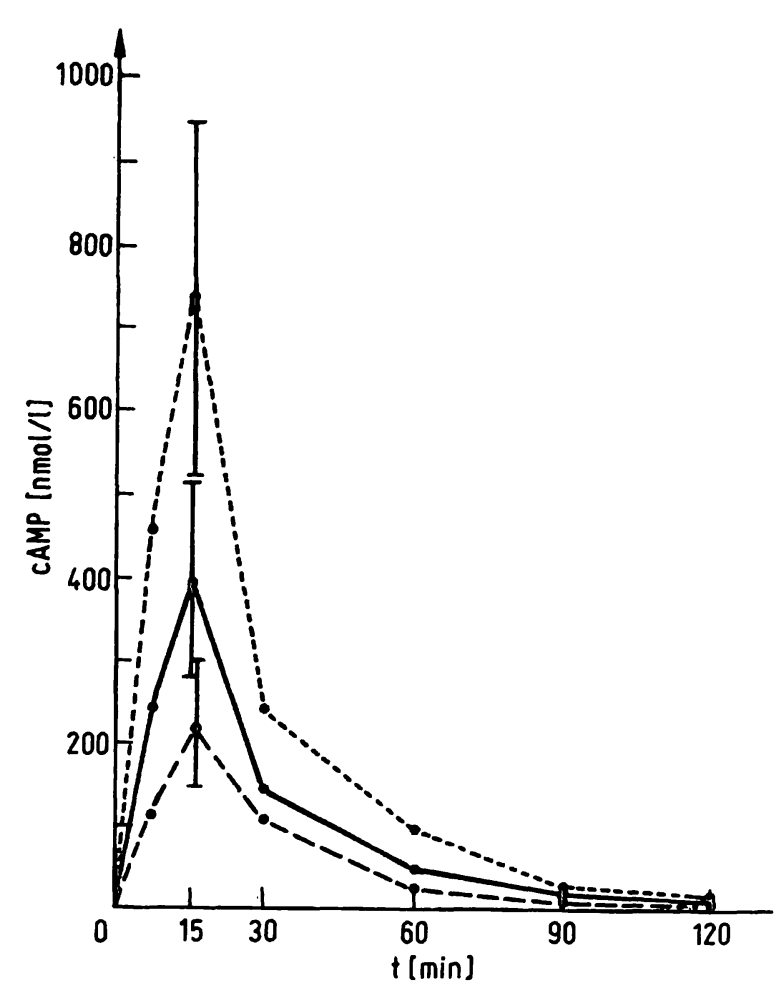

Abb. 2. Zeitlicher Verlauf der Konzentrationen des cyclischen AMP $(\bar{x} \pm s)$ im Plasma nach Injektion von Glukagon bei 7 euthyreoten $(\bullet-\bullet), 7$ hyperthyreoten $(\bullet-\cdots-\cdots)$ und 6 hypothyreoten $(\bullet--\bullet)$ Patienten.

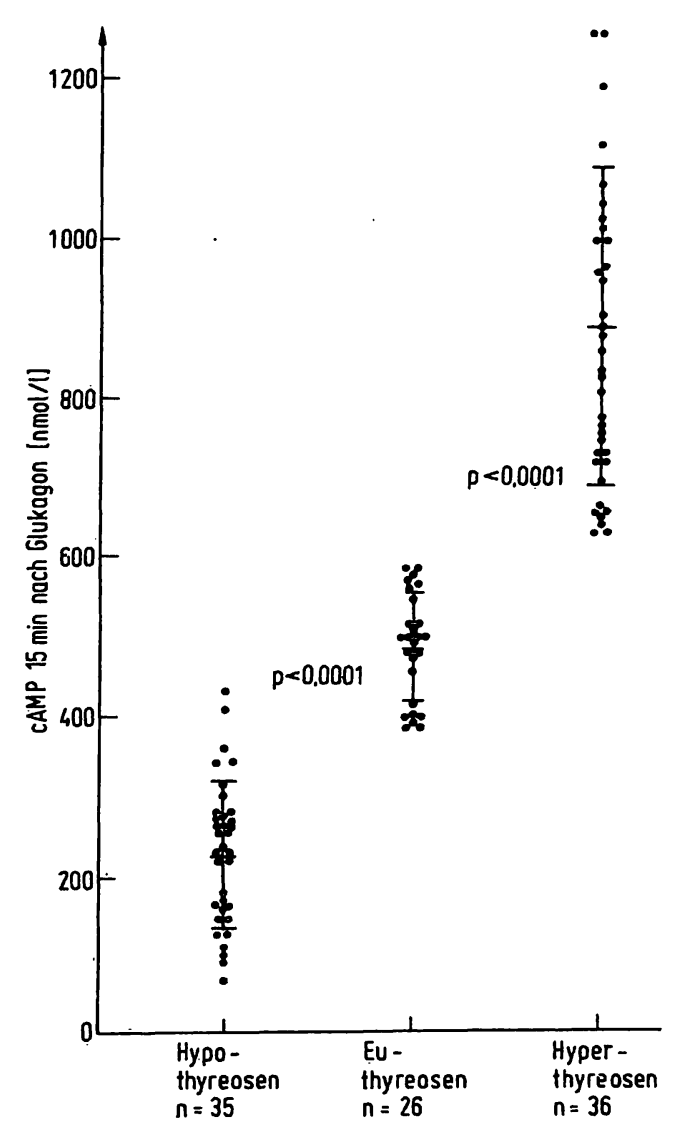

Abb. 3. Mittelwerte mit Standardabweichung sowie Einzelergebnisse der Bestimmung der cAMP-Konzentrationen im Plasma 15 Minuten nach Glukagon-Stimulation bei Normalpersonen, hypo- und hyperthyreoten Patienten. 
Abbildung 4 vergleicht den glukagoninduzierten cAMP. Anstieg im Plasma bei 24 Patienten mit blander Struma mit dem des Normalkollektivs.

Die Patienten mit blander Struma weisen einen 15-Minuten-Wert ( $469 \pm 92 \mathrm{nmol} / \mathrm{l})$ auf, der mit dem des Normalkollektivs vergleichbar ist.

Bei 18 hyperthyreoten Patienten, die thyreostatisch behandelt worden waren, wurde nach Erreichen einer normalen Schilddrüsenfunktion der Glukagon-Test wiederholt. Alle 18 behandelten Patienten wiesen nach $15 \mathrm{Mi}$ nuten eine cAMP-Konzentration auf, die innerhalb des 2s-Bereiches (351-615 nmol/l) des Normalkollektivs lag (Abb. 5).

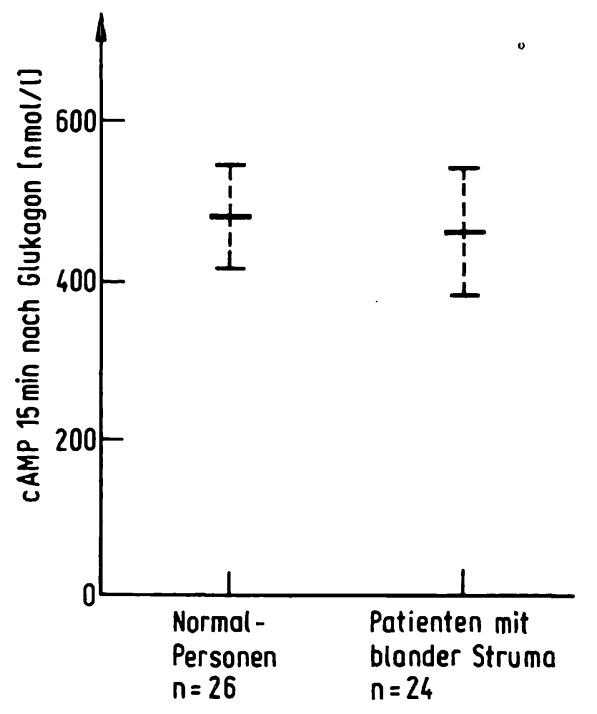

Abb. 4. Mittelwerte und Standardabweichungen der glukagonstimulierten cAMP-Konzentrationen im Plasma bei Normalpersonen und bei Patienten mit blander Struma.

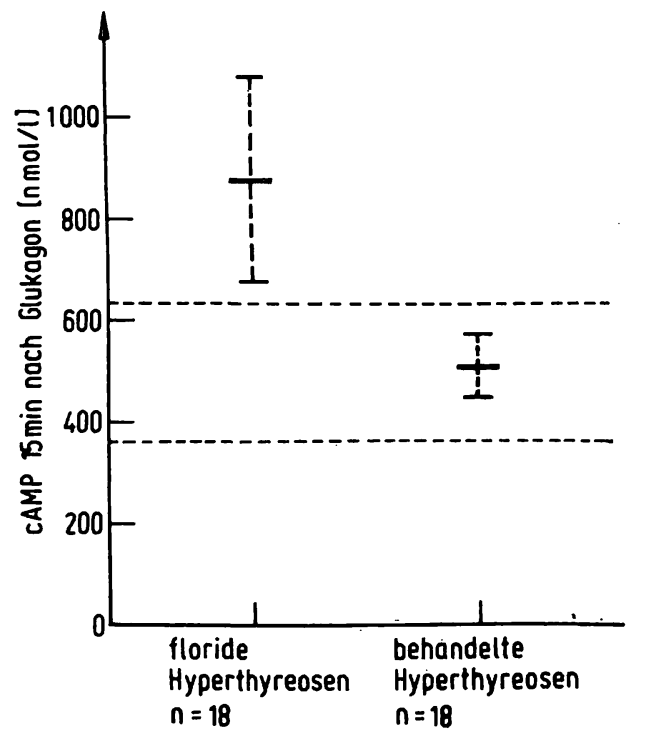

Abb. 5. Mittelwert und Standardabweichung der cAMP-Konzentrationen im Plasma 15 Minuten nach Glukagon-Injektion bei hyperthyreoten Patienten vor und nach thyreostatischer Therapie. Die gepunkteten Linien zeigen den $2 s-B e-$ reich des Normalkollektivs.
Bei allen 7 hypothyreoten Patienten, die nach Substitutionstherapie und Anstieg der Schilddrüsenhormonwerte in den Normbereich erneut getestet wurden, stiegen die Glukagon-stimulierten cAMP-Konzentrationen deutlich höher an als vor der Behandlung und lagen sämtlich innerhalb des 2 s-Bereiches des Normalkollektivs (Abb. 6).

In Abbildung 7 sind die $T_{3}$ - und $T_{4}$-Konzentrationen im Blut sowie das glukagonstimulierte cAMP bei einem Patienten mit einer $T_{3}$-Hyperthyreose unter thyreostatischer Therapie dargestellt. Die Normbereich der $\mathrm{T}_{3}$ - und $\mathrm{T}_{4}$-Konzentrationen sowie der $2 \mathrm{~s}$-Bereich der cAMPKonzentration bei den Normalpersonen sind durch Fettdruck an den Ordinaten gekennzeichnet. Eine Woche

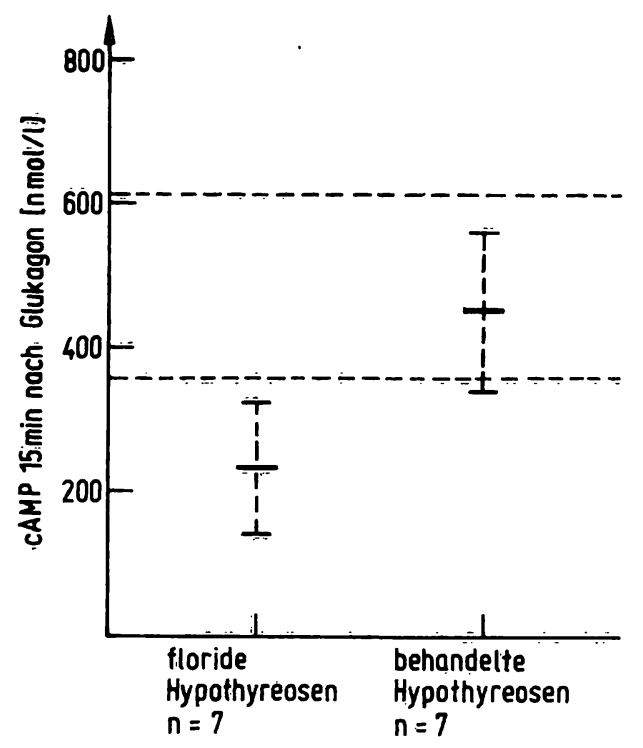

Abb. 6. cAMP-Konzentrationen im Plasma $(\overline{\mathrm{x}} \pm s) 15$ Minuten nach Glukagon-Injektion bei hypothyreoten Patienten vor und nach Substitution von Schilddrüsenhormonen. Die gepunkteten Linien zeigen den 2 s-Bereich des Normalkollektivs.

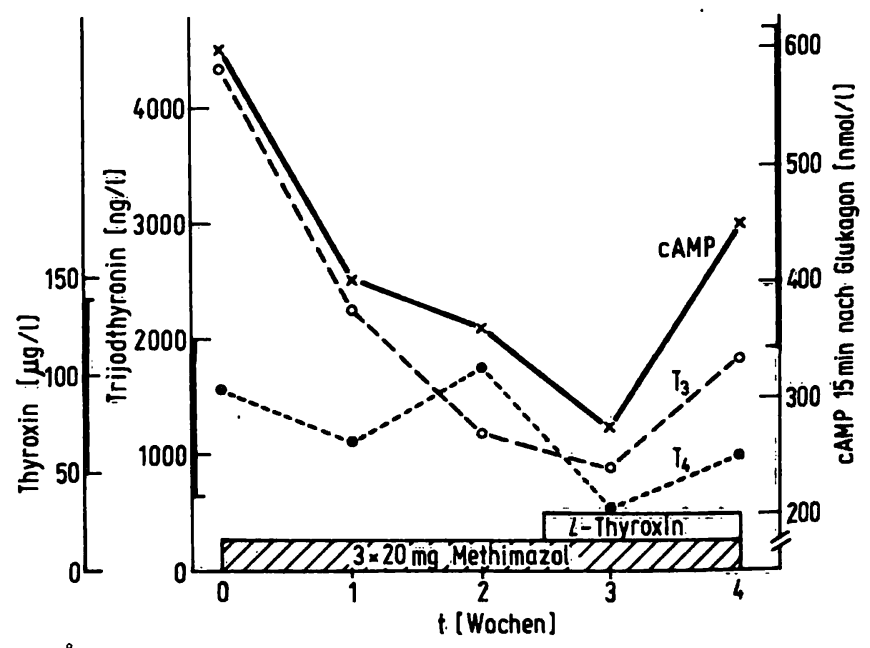

Abb. 7. Konzentrationen von $T_{3}(0--0), T_{4}(\bullet--\cdots)$ und glukagonstimuliertem cAMP $(x-x)$ im Plasma im Behandlungsverlauf einer $T_{3}$-Hyperthyreose. Der Fettdruck an den Ordinaten kennzeichnet den Normbereich. 
nach Therapiebeginn mit $3 \times 20 \mathrm{mg}$ Methimazol ( $\mathrm{Fa}$ vistan $^{\circledR}$ ) sind sowohl das $\mathrm{T}_{3}$ als auch die glukagonstimulierte cAMP-Konzentration bereits deutlich abgesunken. Die cAMP-Konzentration nach 15 Minuten liegt 3 Wochen nach Therapiebeginn entsprechend den subnormalen $\mathrm{T}_{4}$-Werten im hypothyreoten Bereich, steigt jedoch unter der zusätzlichen Therapie mit $100 \mu \mathrm{g} L$-Thyroxin wieder in den Normbereich an.

Zusätzlich wurden 4 Patienten mit dekompensierter Lebercirrhose untersucht. Die glukagonstimulierten cAMPKonzentrationen $(222 \pm 65 \mathrm{nmol} / \mathrm{l})$ lagen bei allen $4 \mathrm{~Pa}$ tienten deutlich unterhalb derjenigen der Normalpersonen.

Bei einem Patienten mit Verschlußikterus fanden wir einen cAMP-Anstieg nach 15 Minuten, der mit $954 \mathrm{nmol} / 1$ weit oberhalb der Werte der Normalpersonen lag.

\section{Diskussion}

Entsprechend der Theorie von Sutherland werden die biologischen Effekte vieler Hormone an ihren Erfolgsorganen dadurch vermittelt, daß nach Bindung des Hormons an einen Rezeptor der Zellmembran und nachfolgender Aktivierung des Enzyms Adenylcyclase zelluläres ATP zu cyclischem AMP umgewandelt wird. Das cAMP bewirkt dann über kettenartig ablaufende Phosphorylierungen eine Änderung der Zellfunktion. Durch das als "second messenger" fungierende cAMP zusammen mit ATP und Proteinkinasen werden Proteine phosphoryliert und damit in die biologisch aktive Form umgewandelt $(10,11)$. Mit einem intrazellulären Anstieg des cAMP steigt auch das cAMP im Plasma an, da die Zellwand für cAMP durchlässig zu sein scheint. Die Konzentrationen im Plasma sind jedoch stets erheblich niedriger als die intrazellulären, so daß die Spezifität der hormonellen Wirkung gewährt bleibt (10). Der Abbau des cAMP erfolgt durch die ubiquitäre Phosphodiesterase, durch die cAMP zu 5'-AMP umgewandelt wird (12). Die Aktivität der Phosphodiesterase kann durch z.B. Coffein, Theophyllin, EDTA gehemmt werden (10). Das cyclische AMP wird glomerulär filtriert, seine Halbwertzeit im Plasma beträgt etwa 30 Minuten, die Plasmaclearance etwa $700 \mathrm{ml} / \mathrm{min}$, wobei etwa $130 \mathrm{ml} / \mathrm{min}$ auf die glomeruläre Filtration und die restlichen $570 \mathrm{ml} / \mathrm{min}$ auf extrarenale Gewebe entfallen (10).

Broadus (13) konnte zeigen, daß verschiedene Hormone die Konzentration von cAMP im Plasma und Urin verändem können. So bewirkt Glukagon einen bis zu 30fachen Anstieg der Konzentration im Plasma und einen vergleichbar großen Anstieg der Konzentration im Harn. Durch die Gabe von Calcitonin und $\beta$-Rezeptorenstimulatoren kann die cAMP-Konzentration im Plasma auf das 2-3-fache des Ausgangswertes gesteigert werden.

Daß Glukagon die Bildung von CAMP in der Leber stimuliert, konnten Rall et al. (1) bereits 1957 nachwei- sen. Markmann (14), Robinson (15) und Exton (16) kamen zu dem gleichen Ergebnis. Broadus (3) infundierte 1970 erstmals Glukagon zur cAMP-Bestimmung beim Menschen (25-200 mg/min · kg Körpergewicht) und Elkeles (5) injizierte 1974 eine konstante, einmalige Dosis von $1 \mathrm{mg}$.

Auch wir entschieden uns in Ubereinstimmung mit anderen Arbeitsgruppen für die Menge von $1 \mathrm{mg}$ Glukagon nach entsprechenden Vorversuchen mit $0,5 \mathrm{mg}$, die offensichtlich bei adipösen Patienten für eine optimale Stimulation nicht ausreichte. Durch Voruntersuchungen mit Blutentnahmen nach 7,5, 15, 30,60, 90 und $120 \mathrm{Mi}$ nuten konnten wir zeigen, daß das cAMP 15 Minuten nach Glukagoninjektion ein Maximum erreicht (Abb. 2), so daß bei späteren Versuchen auf die anderen Entnahmen verzichtet wurde. Dies stimmt mit den Ergebnissen von Elkeles (5), Liljenquist (18), Lazarus (7) und Guttler (9) überein.

Karlberg (19) wies 1974 auf signifikante Unterschiede in den basalen Konzentrationen des cAMP im Plasma zwischen Hypothyreoten, Normalpersonen und Hyperthyreoten hin. Unsere Untersuchungen an einem erheblich gröBeren Kollektiv konnten dies - wie auch die meisten der anderen in der Folgezeit erschienenen Arbeiten (5-9) nicht bestätigen.

Hinweise darauf, daß der glukagoninduzierte cAMP-Anstieg durch Schilddrüsen-Hormone beeinflußt wird, ergaben sich 1973 erstmals durch die Arbeit von Campbell \& Kane-Maguire (4). Diese Autoren konnten mit Versuchen an isolierten Rattenleber-Organkulturen nachweisen, daß der glukagoninduzierte cAMP-Anstieg durch eine Präinkubation mit $T_{3}$ und $T_{4}$ gesteigert werden kann. Elkeles (5) untersuchte daraufhin erstmals die naheliegende Frage, ob der glukagoninduzierte cAMPAnstieg im Plasma des Menschen von der Schilddrüsenfunktion abhängig ist. Er fand die cAMP-Reaktion auf Glukagon bei hypothyreoten Patienten abgeschwächt und bei Hyperthyreoten gesteigert im Vergleich zu Normalpersonen.

In der Folgezeit untersuchten mehrere Autoren entsprechende Kollektive mit der gleichen Fragestellung.

Guttler (6) führte den Test 1975 an 4 hypo- und 4 hyperthyreoten Patienten sowie 5 Normalpersonen durch. Dabei fand er - im Gegensatz zu Elkeles - bei den Hypothyreoten glukagoninduzierte cAMP-Konzentrationen, die denen des Normalkollektivs entsprachen. Die Hyperthyreoten zeigten auch bei Guttler erhöhte 15-Minuten-Werte für cAMP.

Lazarus (7) testete 197620 hypo-, 7 hyper- sowie 19 euthyreote Patienten.Madsen (8) stellte 1977 Kollektive von 14 Hypo- und 13 Hyperthreoten einer Gruppe von 17 Normalpersonen gegenüber. Beide Autoren bestätigten Elkeles' Ergebnis, indem sie bei Patienten mit Hypothyreose erniedrigte und bei solchen mit Hyper- 
thyreose im Plasma erhöhte cAMP-Konzentrationen 15 Minuten nach Glukagon-Injektion nachwiesen.

Lazarus untersuchte sein Kollektiv - nach Therapie und Erreichen einer normalen Schilddrüsenfunktion - erneut. Er fand 15-Minuten-Werte für cAMP, die denen des Normalkollektivs vergleichbar waren. Auch Madsen testete 2 hypo- und 6 hyperthyreote Patienten nach durchgefuihrter Therapie erneut und fand jetzt bei den ursprünglich Hypothyreoten glukagoninduzierte cAMP-Konzentrationen, die denen des Normalkollektivs entsprachen, während er bei den Hyperthyreoten eine gewisse Trägheit in der Abnahme einer einmal induzierten Hyperreaktion des CAMP auf Glukagon feststellte.

Wir konnten in unserer Arbeit mit größeren Kollektiven signifikante Unterschiede in der Reaktion des CAMP im Plasma auf Glukagon zwischen Hyper- und Hypothyreosen sowie Normalpersonen nachweisen.

Während die hyperthyreoten Patienten einen deutlich stärkeren cAMP-Anstieg im Plasma als die Normalpersonen zeigten, war der der Hypothyreosen signifikant niedriger als der des Vergleichskollektivs, allerdings traten hier Überlappungen der Einzelwerte mit dem Normalbereich auf.

Von den hyperthyreoten Patienten, bei denen wir nach thyreostatischer Therapie und Erreichen einer normalen Schilddrüsenfunktion den Glukagon-Test wiederholten, wiesen alle 18 nach 15 Minuten einen cAMP-Anstieg auf, der demjenigen der Normalpersonen entsprach.

Bei allen 7 hypothyreoten Patienten, die wir nach Substitutionstherapie und Anstieg der SchilddrüsenhormonWerte in den Normbereich erneut testeten, hatten sich die glukagonstimulierten cAMP-Konzentrationen normalisiert. Auch bei den Patienten mit einer blanden Struma fanden wir nach Glukagon-Injektion cAMP-Werte, die denen der Normalpersonen vergleichbar waren. Dies erklärt sich durch die klinisch und laborchemisch normale Schilddrüsenfunktion dieser Patienten.

Folgende Modellvorstellungen versuchen den - in Abhängigkeit von der Schilddrüsenfunktion - unterschiedlichen cAMP-Anstieg nach Glukagongabe zu erklären:

1. Schilddrüsenhormone bewirken über eine Hemmung der Phosphodiesterase - und somit über einen verminderten Abbau des cycl. $3^{\prime}: 5^{\prime}$-AMP - eine erhöhte cAMPKonzentration. Dies wurde zunächst von Mandel (20) 1967 aufgrund von in-vitro Versuchen angenommen. Krishna (21), Challoner \& Allen (22) und Caldwell \& Fain (23) fanden allerdings normale PhosphodiesteraseKonzentrationen bei hyperthyreoten Patienten, so daß die gesteigerte cAMP-Antwort auf Glukagon wahrscheinlich nicht durch eine verminderte Phosphodiesterase-Aktivität erklärt werden kann.

2. Schilddrüsenhormone beeinflussen die Anzahl der Glukagon-Rezeptoren und bewirken über deren Vermehrung eine Steigerung der Glukagon-Empfindlichkeit. Vor allem Madsen (8) favorisiert diese Vorstellung und sah sich unterstützt durch die Ergebnisse von Krishna (21), nach denen sich die Adenylcyclase-Aktivität selbst nicht mit der Schilddrüsenfunktion ändert.

Guttler erwähnt in seiner Arbeit aus dem Jahre 1975 (6) weitere denkbare Vorstellungen, für die bisher aber noch keine konkreten Überprüfungen vorliegen:

3. Schilddrüsenhormone steigern die Hormon-RezeptorAffinität.

4. Schilddrüsenhormone steigern die ATP-Freisetzung am Ort der Konversion zu cAMP.

5. Schilddrüsenhormone stimulieren einen Übermittler in der Zellwand, wie ihn Rodbell (24) für das GTP beschrieben hat.

6. Schilddrüsenhormone steigern die Membran-Permeabilität für cAMP.

Broadus (3) konnte 1970 durch Versuche mit Glukagonperfundierten Rattenlebern und an hepatektomierten Hunden nachweisen, daß die Leber die Hauptquelle des cAMP-Anstiegs nach Glukagon-Injektion darstellt. Dies wurde 1974 durch Untersuchungen von Lilienquist (18) mittels Blutentnahmen aus der Arteria brachialis und einer Lebervene beim Menschen bestätigt.

Aufgrund der genannten Ergebnisse ist zu postulieren, daßß bei Patienten mit einer Leberinsuffizienz der Anstiêg des cAMP nach Glukagon-Injektion geringer ausfâllt.

In Ubereinstimmung mit anderen Autoren $(25,8)$ fanden auch wir bei 4 Patienten mit dekompensierter Lebercirrhose einen deutlich geringeren Anstieg des cAMP als bei Normalpersonen. Dies bestätigt somit die oben zitierten Arbeiten von Broadus und Liljenquist.

Bei einem Patienten mit einem extrahepatischen Verschlußikterus lag die glukagonstimulierte cAMP-Konzentration - trotz subnormaler $\mathrm{T}_{3}$ - und $\mathrm{T}_{4}$-Werte deutlich oberhalb des Normbereiches. Dieses Ergebnis stimmt mit den Resultaten von Davies (25) und Francavilla (26) überein und ist möglicherweise durch eine verminderte cAMP-Ausscheidung über die Gallenwege bei extrahepatischem Verschluß erklärbar.

Verschiedene Pharmaka können die Konzentration des cAMP im Plasma bzw. die cAMP-Antwort auf GlukagonInjektion beeinflussen. So zeigten Liddle \& Hardman 1971 (10) eine Phosphodiesterase-Hemmung durch Coffein und Theophyllin, welche eine Verstärkung der cAMP-Reaktion auf Glukagon zur Folge hat.

Diese Autoren wiesen in ihrer Arbeit außerdem auf eine Abnahme des cAMP-Gehaltes in Leber und Fettgewebe durch Insulin hin.

Ball (27) stellte fest, daß auch $\beta$-Rézeptor-Stimulatoren wie Isoproteronol, Adrenalin und Noradrenalin einen Anstieg des CAMP im Plașma hervorrufen.

DeRubertis (28) zeigte 1974, daß die Próstaglandine $\mathrm{E}_{1}$ und $\mathrm{E}_{2}$ einen leichten Anstieg des cAMP auslösen; mit 
Glukagon gemeinsam infundiert, vermindern sie aber die glukagoninduzierte cAMP-Reaktion. Der Autor vermutet ein gemeinsames Adenylcyclase-System für Glukagon und die Prostaglandine, wobei letztere als Regelkomponente eine hemmende Funktion bezüglich der Wirkung von Glukagon auf die Leber haben könnten.

Aufgrund unserer Untersuchungsergebnisse stellt der Glukagontest ein einfaches und verläßliches Verfahren dar, das Rückschlüsse auf die Wirkung von Schilddrüsenhormonen an peripheren Geweben ermöglicht. Einschränkend muß jedoch gesagt werden, daß die Aussagefähigkeit des Testes bei schweren Leber- und Gallenwegserkrankungen begrenzt ist. $\mathrm{Ob}$ dem Test eine größere klinische Bedeutung zukommen kann, läßt sich erst nach Untersuchungen an Patienten, die eine erhebliche Diskrepanz zwischen klinischem Bild und Ergebnis der laborchemischen Bestimmungen der Schilddrüsenhor-
mon-Konzentrationen aufweisen, beantworten. Dabei ist mit diesem Test besonders eine Abgrenzung zwischen normaler und hyperthyreoter Stoffwechsellage möglich, während sich bei Hypothyreosen Uberlappungen mit dem Normbereich ergeben können. Die routinemäßige Bestimmung von Serum- $T_{3}$ und $-T_{4}$ sowie den Thyroliberin (TRH)-Test kann der Glukagon-Test wegen mangelnder Spezifität sicher nicht ersetzen. Er stellt aber, wie andere Kenngrößen (Messung der systolischen Zeiten, der Achillessehnenreflexzeit, der Dopamin- $\beta$-hydroxylase und Kreatinkinase-Aktivität, des Natriumgehaltes der Erythrocyten, der Konzentration an Geschlechtshormon-bindendem Globulin und Cholesterin im Serum, sowie der Thyrotropin-Antwort nach Thyroliberin-Gabe) einen weiteren Schritt in der diagnostisch dringend erforderlichen Uberprüfung der direkten Wirkung von Schilddrüsenhormonen an ihren Erfolgsorganen, den peripheren Geweben, dar.

\section{Literatur}

1. Rall, T. W., Sutherland, E. W. \& Berthet, J. (1957), J. Biol. Chem. 224, 463-475.

2. Sutherland, E. W. \& Robison, G. A. (1966), Pharmacol. Rev. $18,145-161$.

3. Broadus, A. E., Kaminsky, N. I., Northcutt, R. C., Hardman, J. G., Sutherland, E. W. \& Liddle, G. W. (1970), J. Clin. Invest. 49, 2237-2245.

4. Campbell, A. K. \& Kane-Maguire, B. (1973), Biochem. Soc. Transact. $1,1002-1005$.

5. Elkeles, R. S., Lazarus, J. H., Siddle, K. \& Campbell, A. K. (1975), Clin. Sci. Mol. Med. 48, 27-31.

6. Guttler, R. B., Otis, C. L., Shaw, J. W., Warren, D. W. \& Nicoloff, J. T. (1975), Thyroid Horm. Metab. Proc. Int. Symp. 1974 (Harland, A. \& Orr, J. S., eds.), 201-211.

7. Lazarus, J. H., Shetty, K. J. \& Siddle, K. (1976), Excerpta Med. Int. Congr. Ser. (Thyroid Res.) 378, 606-609.

8. Madsen, S. N. (1977), Acta Endocrinol. (Kbh.) 85, 760-768.

9. Guttler, R. B., Croxson, M. S., DeQuattro, V. L., Warren, D. W., Otis, C. L. \& Nicoloff, J. T. (1977), Metabolism 26, 1155-1162.

10. Liddle, G. W. \& Hardman, J. G. (1971), New Engl. J. Med. $285,560-566$

11. Reimann, E. M., Brostrom, C. O., Corbin, J. D., King, C. A. \& Krebs, E. G. (1971), Biochem. Biophys. Res. Comm. 42, 187-194.

12. Butcher, R. W. \& Sutherland, E. W. (1962), J. Biol. Chem. $237,1244-1250$.

13. Broadụs, A. E., Hardman, J. G., Kaminsky, N. I., Ball, J. H., Sutherland, E. W. \& Liddle, G. W. (1971), Ann. N. Y. Acad. Sci. 185, 50-66.

14. Makman, M. H. \& Sutherland, E. W. (1964), Endocrinology $75,127-134$.
15. Robison, G. A., Exton, J. H., Park, C. R. \& Sutherland, E. W. (1967), Fed. Proc. 26, 257 (Abstr. 16).

16. Exton, J. H. \& Park, C. R. (1968), Adv. Enzyme Regul. 6, $391-405$.

17. Dich, J. \& Gluud, C. N. (1976), Acta Physiol. Scand. 97, 457-469.

18. Liljenquist, J. E., Bomboy, J. D., Lewis, S. B., SinclairSmith, B. C., Felts, P. W., Lacy, W. W., Crofford, O. B. \& Liddle, G. W. (1974), J. Clin. Invest. 53, 198-204.

19. Karlberg, B. E., Henriksson, K. G. \& Andersson, R. G. G. (1974), J. Clin. Endocrinol. Metab. 39, 96-101.

20. Mandel, L. R. \& Kuehl, F. A. (1967), Biochem. Biophys. Res. Comm. 28, 13-18.

21. Krishna, G., Hynie, S. \& Brodie, B. B. (1968), Proc. Nat. Acad. Sci. USA 59, 884-889.

22. Challoner, D. R. \& Allen, D. O. (1970), Metabolism 19, 480-487.

23. Caldwell, A. \& Fain, J. N. (1971), Endocrinology 89, 1195-1204.

24. Rodbell, M., Lin, M. C. \& Salomon, Y. (1974), J. Biol. Chem. 249, 59-65.

25. Davies, T. F., Prudhoe, K. \& Douglas, A. P. (1976), Brit. Med. J. 1, 931-933.

26. Francavilla, A., Pansini, F., Sansone, F., Albano, O. \& Martellotta, G. (1977), Clin. Chim. Acta 75, 351-357.

27. Ball, J. H., Kaminsky, N. I., Hardman, J. G., Broadus, A. E., Sutherland, E. W. \& Liddle, G. W. (1972), J. Clin. Invest. $51,2124-2129$.

28. DeRubertis, F. R., Zenser, T. V. \& Curnow, R. T. (1974), Endocrinology 95, 93-101.
Prof. Dr. med. J. Herrmann Medizinische Klinik C Universität Düsseldorf Moorenstraße 5 D-4000 Düsseldorf 


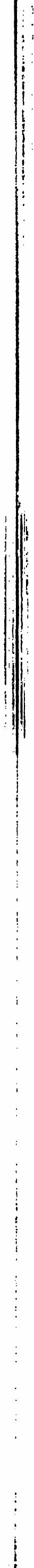

